\title{
Optimization of structure of the wide band interference filters
}

\author{
Alexandr Mitsa, József Holovács, Vasil Petcko \\ Uzhgorod National University
}

\begin{abstract}
Optimal parameters of some wide band interference filters have been investigated and described the most effective constructions of multidimensional search methods for the given problem have been defined. Features of realization of multidimensional search methods are also described.

Keywords: modeling, optimization, multidimensional search methods, light transmission, multilayer interference coating, the wide band interference filters.
\end{abstract}

\section{Introduction}

Multilayer optical coatings are successfully employed in various fields, such as optical and scientific instrumentation manufacturing, astronomy, spectroscopy, medicine, etc. A great number of laboratories and experts are involved in designing and manufacturing modern optical coatings [1-11].

\section{Modeling and optimization}

To estimate the influence of changing interference systems parameters on the resulting transmission the matrix method was used. It based on the determination of a characteristic matrix [2]. The matrix method for calculating spectral coefficients of the layered media was first suggested by F. Abeles (1950) and has been widely employed ever since. The results of the previous section allow to provide here its very simple presentation. Let us assume a multilayer coating consisting of a finite number of homogeneous and isotropic layers.

If the geometrical thickness of a layer is equal to $d$, and refraction coefficient is equal to $n$, the characteristic matrixes of the homogeneous dielectric film have the appearance: 


$$
M(n, d, \lambda)=\left\|\begin{array}{cc}
\cos \delta(n, d, \lambda) & -(i / p) \sin \delta(n, d, \lambda) \\
-i p \sin \delta(n, d, \lambda) & \cos \delta(n, d, \lambda)
\end{array}\right\|,
$$

where $\delta(n, d, \lambda)=2 \pi n d \cos \theta / \lambda$ is the phase thickness of a layer, $p=\sqrt{\varepsilon / \mu \cos \delta}$. In the case when the direction of propagation of radiation coincides with a perpendicular to the interface, $\delta=0$ and correspondingly $p=n$.

Knowing a characteristic matrix of one layer (2.1), we can determine a characteristic matrix of $k$-th layer of multilayer systems, as a product of matrixes of each layer:

$$
M(\bar{n}, \bar{d}, \lambda)=M_{k}\left(n_{k}, d_{k}, \lambda\right) \cdot M_{k-1}\left(n_{k-1}, d_{k-1}, \lambda\right) \cdots M_{1}\left(n_{1}, d_{1}, \lambda\right),
$$

where $M_{j}$ is a characteristic matrix of $j$-th layer; $\bar{n}=\left(n_{1}, n_{2}, \ldots, n_{k}\right)$ is a vector of the values of refraction indices of layers; $\bar{d}=\left(d_{1}, d_{2}, \ldots, d_{k}\right)$ is a vector of geometrical thicknesses of layers.

From (2.2) it is easy to find a value of a MLS transmission at the fixed values of $\bar{n}, \bar{d}$ and $\lambda$ :

$$
\begin{aligned}
& T(\bar{n}, \bar{d}, \lambda)= \\
& 1-\left[\left\lfloor\frac{\left.n_{0}\left(M_{11}(\bar{n}, \bar{d}, \lambda)+n_{s} M_{12}(\bar{n}, \bar{d}, \lambda)\right)-n_{s} M_{22}(\bar{n}, \bar{d}, \lambda)+M_{21}(\bar{n}, \bar{d}, \lambda)\right)}{\left.n_{0}\left(M_{11}(\bar{n}, \bar{d}, \lambda)+n_{s} M_{12}(\bar{n}, \bar{d}, \lambda)\right)-n_{s} M_{22}(\bar{n}, \bar{d}, \lambda)+M_{21}(\bar{n}, \bar{d}, \lambda)\right)}\right]^{2}\right.
\end{aligned}
$$

where $n_{0}, n_{s}$ are the refraction indices of external environment and substrate accordingly $M_{11}, M_{12}, M_{21}, M_{22}$ are the elements of a characteristic matrix $M_{j}$.

For the numerical calculation of the transmittance spectra of MLS the objective function is represented as [3]:

$$
\max _{\bar{n}, \bar{d}} F(\bar{n}, \bar{d})=\left(\frac{1}{L} \sum_{i=1}^{L} T^{2}\left(\bar{n}, \bar{d}, \lambda_{i}\right)\right)^{1 / 2},
$$

where $L$ is a number of a grid points for a spectral interval from $\lambda_{1}$ to $\lambda_{2}$. At the it's uniform distribution with a step $\Delta \lambda$

$$
L=\frac{\lambda_{2}-\lambda_{1}}{\Delta \lambda}+1
$$

where $\lambda_{1}$ and $\lambda_{2}$ are the short-wave and the long-wave boundary accordingly of researched wave spectral region.

In our experience various gradient methods are efficient for a search of the merit function minimum. All the methods of the type imply re-iterated calculations of the merit function and its gradient, i.e., the vector comprised by partial derivatives of the merit function with respect to the layers sought for parameters. These two operations are essential, and so the rate and precision of their fulfillment determine to a considerable degree the calculation potentials of the method [6].

Very often multilayer optics synthesis problems are solved success-fully through reiterated optimization of the merit function with series of random starting designs. 
As a quasioptimal solution, a vector of the coating parameters is taken, which corresponds to the deepest of the obtained minima. Sometimes, it is worthwhile to pick out several quasioptimal solutions, especially, if the minima are about the same depths. The final selection of the best solution can be done on the basis of the results of their practical implementation.

It is difficult to give all-embracing recommendations concerning the starting design choice and algorithm peculiarities for different types of synthesis problems. Let us only note here that good starting designs can be also set in case of wide-band mirrors and polarizers synthesis [6-7].

Usually, the steepest descent method predetermines a fast de-crease of the function along the first few directions of one-dimension, minimizations. Then the speed of the decrease slows radically. This is especially evident when the function isolines have a "ravine-like" structure. Experience shows that the merit function isolines in the synthesis problems look exactly like the former. The "ravine-like" pattern of the merit function grows fast as the number of the F-function variables, i.e., the number of the layers increases. The conjugate gradients method allows to manage the ravine-like pattern of the minimized function in a simple and reliable way. The conjugate gradients method differs from the steepest descent one in the fact that a one-dimension minimization is not implemented along the antigradient but rather along some "adjusted" direction obtained with the previous descent direction taken into account $[1,3,6]$.

Let us now discuss synthesis problems where the starting design choice is vague, as a rule. Such are the problems of synthesis of antirefrection coatings, neutral beam splitters, multilayer systems featuring non-standard spectral properties. Let us make another remark concerning optimization of multiextremal functions. In principle, there are mathematical methods of their global minimum search. However, a strict employment of these methods which would ensure determining the global minimum is not feasible practically when the number of variables exceeds 1 or 2 because it implies the necessity of tremendous amounts of computer time. Due to this fact, methods are mostly used which, speaking strictly mathematically, cannot guarantee unconditional determining of the global minimum, but they are completely justifiable in solving practical problems. The considerations above, related to a search of quasioptimal solution of the synthesis problems accord with this approach [3].

\section{Result}

Recently in [12] was proposed two components wide band interference filters for visible spectral range. Bat on spectral dependence of transmitivity there are some deviation from ideal curve (Figure 1, curve 1). In order to improve level of transmitivity in the visible range up to $100 \%$ we applied different methods of multidimensional search. During optimization procedure we have found best parameters compare with [12] for antireflecting two component multinary optical coatings.

We used in parallel methods on p processors. In this case the acceleration in 


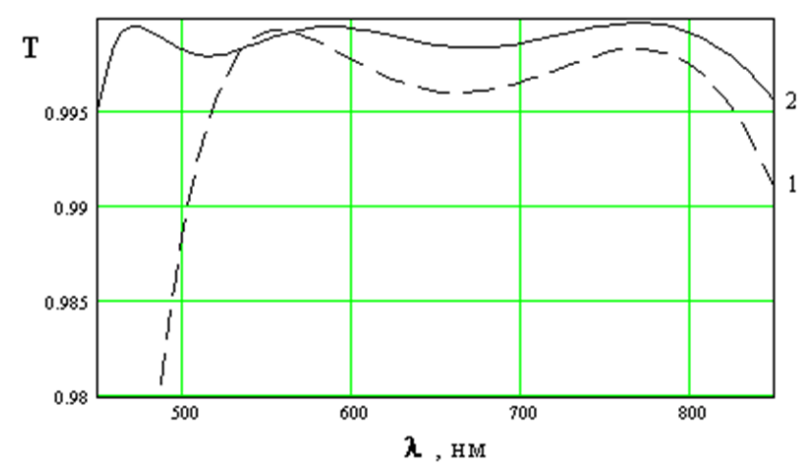

Figure 1: Coefficient of transmittivity for 7 layers two components filters with low $(\mathrm{L})$ and high $(\mathrm{H})$ refractive indexes $n_{L}=1.35$, $n_{H}=2.1$, substrate $n_{s}=1.52$ :

1 - optical thicknesses for working wavelength $\lambda_{0}$ are equals 0.06 :

$0.02: 0.35: 0.02: 0.07: 0.42: 0.21$ taken from [12];

2 - optical thicknesses for working wavelength $\lambda_{0}$ are equals 0.038 : $0.035: 0.047: 0.126: 0.014: 0.059: 0.155$ after optimization, this work

time range is possible compare to one processor computer. In linear approximation this acceleration is approximately is as integral part of $q=[k / p+1]$. On Figure 1 curve 2 is ease seen that optimization parameters which we got are improve spectral dependents of transmitivity compare with results, obtained in [12].

In solving any kind of the synthesis problems, it is necessary to take into account a number of other non-quantitative aspects, including, firstly, the necessary costs of the solution in terms of computer time and labors costs. A waste of many hours in search of an optimal solution is evidently senseless if, on the other hand, we can find within several minutes "an acceptably adequate" solution satisfying all the requirements. All the above causes the following conclusion concerning the merit function optimization: a global optimization is frequently redundant and it is advisable to look for a sufficiently thorough local minimum ensuring the required accuracy of the pre-set spectral dependence approximation and good feasibility properties of the coating design. Such "quasioptimal" solution is frequently achieved due to a good selection of a starting design for the merit function optimization. The researcher's personal experience is undoubtedly of utmost importance, though a number of common rules can be recommended. This, firstly, concerns, edge filters of various type. It is advisable to use quarter-wave mirrors or some combinations of such mirrors as the first approximation to their design.

This method is useful for a wide range of experts from specialists of practical applications to specialists elaborating the theory of thin film coatings. Using this method it is possible to receive results which are easily put into practice. 


\section{References}

[1] Sh. Furman and A.V.Tikhonravov, "Basics of optics of multilayer systems", Editions Frontiers, Gif-sur Yvette, 1992, 242 p.

[2] Abeles F. Matrix method / Ann.de Physique. 1950. V.5. P. 596-640.

[3] Stetsyuk P. I., Mitsa A. V. Parameter Optimization Problems for Multilayer Optical Coatings, Cybernetics and Systems Analysis, Volume 41, Issue 4, Jul 2005, Pages $564-571$.

[4] Rabinovich K., Pagis A. Multilayer Antireflection Coatings: Theoretical Model and Design Parameters // Appl. Opt., 1975, v. 14, No. 6. P. 1326-1334.

[5] Apparao K. V. S. R., An Improved Optimization Method of Designing Thin Film Filters. - Indian J. Pure Appl. Phys., 1975, v. 13, No. 3. P. 183-186.

[6] Tikhonov A.N., Tikhonravov A.V., Trubetskov M.K. Second oder optimization methods in the synthesis of multilayer coatings // Comp. Maths. Math. Phys., Vol. 83, 1993. P. 1339-1352.

[7] Tikhonravov A.V. Some theoretical aspects of thin film optics and their applications // Applied Optics, 1993, Vol. 32, No. 28. P. 5417-5426.

[8] Jakovlev P.P., Meshkov B.B. Desining of interference coatings. Moskva. Mechanical engeneering. 1987. (In Russian)

[9] Born M., Wolf E. Priciples of optics. 4 ed. Pergamon Press. Oxford 1970.

[10] Pervak Y., Mitsa O., Holovach J., Fekeshgazi I. Influence of transition film-substrate layers on optical properties of multilayer structure // The International Society for Optical Engineering. Vynnytsa. 2000. Vol. 4425.

[11] Holovacs J., Mitsa A., Mitsa V. Computer modelling of characteristics of structures with short periods //Proc. 4th International Conference on Applied Informatics. Eger-Noszvaj. Hungary. 1999. P. 51-57 .

[12] Vedenskij V.D., Stolov E.G., Metelnikov A.A., Furman Sh.A., Semin E.G. Wide band optical coating. Patent No. 838629 (former USSR), MKI G 02 B 5/28. Published 15.10.79, Bulletin No. 22. 